GIGABAR MATERIAL

PROPERTIES EXPERIMENTS

ON NIF AND OMEGA

D. C. Swift, J. A. Hawreliak, D. Braun, A. Kritcher, S. Glenzer, G. W. Collins, S. D. Rothman, D. Chapman, S. Rose

August 9, 2011

American Physical Society Topical Conference on Shock Compression of Condensed Matter Chicago, IL, United States June 26, 2011 through July 1, 2011 
This document was prepared as an account of work sponsored by an agency of the United States government. Neither the United States government nor Lawrence Livermore National Security, LLC, nor any of their employees makes any warranty, expressed or implied, or assumes any legal liability or responsibility for the accuracy, completeness, or usefulness of any information, apparatus, product, or process disclosed, or represents that its use would not infringe privately owned rights. Reference herein to any specific commercial product, process, or service by trade name, trademark, manufacturer, or otherwise does not necessarily constitute or imply its endorsement, recommendation, or favoring by the United States government or Lawrence Livermore National Security, LLC. The views and opinions of authors expressed herein do not necessarily state or reflect those of the United States government or Lawrence Livermore National Security, LLC, and shall not be used for advertising or product endorsement purposes. 


\title{
GIGABAR MATERIAL PROPERTIES EXPERIMENTS ON NIF AND OMEGA
}

\author{
Damian C. Swift*, James A. Hawreliak ${ }^{\dagger}$, David Braun ${ }^{\dagger}$, Andrea Kritcher ${ }^{\dagger}$, Siegfried \\ Glenzer $^{\dagger}$, Gilbert Collins ${ }^{\dagger}$, Steven D. Rothman ${ }^{* *}$, David Chapman ${ }^{* *}$ and Steven Rose ${ }^{\ddagger}$ \\ *PLS-CMMD, Lawrence Livermore National Laboratory, 7000 East Avenue, Livermore, CA 94551, U.S.A. \\ ${ }^{\dagger}$ Lawrence Livermore National Laboratory, 7000 East Avenue, Livermore, CA 94551, U.S.A. \\ ${ }^{* *}$ Atomic Weapons Establishment, Aldermaston, Reading, RG7 4PR, U.K. \\ Department of Physics, Imperial College, London, SW7 2AZ, U.K.
}

\begin{abstract}
The unprecedented laser capabilities of the National Ignition Facility (NIF) make it possible for the first time to countenance laboratory-scale experiments in which gigabar pressures can be applied to a reasonable volume of material, and sustained long enough for percent level equation of state measurements to be made. We describe the design for planned experiments at the NIF, using a hohlraum drive to induce a spherically-converging shock in samples of different materials. Convergence effects increase the shock pressure to several gigabars over a radius of over 100 microns. The shock speed and compression will be measured radiographically over a range of pressures using an x-ray streak camera. In some cases, we will use doped layers to allow a radiographic measurement of particle velocity.
\end{abstract}

Keywords: shock, equation of state, laser

PACS: 07.35.+k, 71.00.00, 91.45.Bg, 91.60.Gf

\section{INTRODUCTION}

Pressures in the gigabar $(100 \mathrm{TPa})$ regime are predicted to occur in the cores of massive exoplanets $[1,2]$. Besides helping to interpret the structure of exoplanets, equations of state (EOS) in this regime are important in the study of brown dwarf formation, and thus to guide estimates of non-luminous mass given the observable stars, which is necessary to determine whether new physics is needed to explain galactic rotation curves and thus the existence of exotic dark matter [3]. Technologically, these pressures and higher occur in the implosion of thermonuclear fuel capsules, and EOS are therefore relevant in the development of inertial confinement fusion.

Pressures over a few megabars (100 GPa) are too high to be induced using static laboratory techniques such as diamond anvil cells, and must be generated and studied in a transient way by shock or ramp load- ing. In the past, nuclear explosions have been used to induce shocks of pressures up to several gigabars by ablation [4,5], but few such measurements have been made. Laser-induced ablation on $\sim 10 \mathrm{~kJ}$ class lasers such as Omega is now fairly routine for the generation of pressures up to several megabars, with direct laser irradiation of an ablator or laser heating of an $\mathrm{x}$ ray hohlraum [6]. The equivalent experiments at the megajoule class NIF have demonstrated pressures of $\sim 5 \mathrm{TPa}$ at less than full energy [7].

On any facility, the maximum pressure that can be induced depends on the power available and the volume into which it can be delivered. For a useful EOS measurement, a sufficient volume of matter must be prepared in the high pressure state and preserved long enough for a measurement to be made, thus in practice the maximum pressure for a useful experiment depends also on the total energy available. Many laser platforms are capable of preparing 
matter at high pressures and temperatures, but the volume and duration required for measurements of reasonable accuracy are extremely challenging. Even with the megajoule energies available at NIF, pressures induced by ablation are unlikely to exceed a couple of hundred megabars for EOS experiments.

Converging shocks have been used previously to increase the pressure available from chemical explosives from a few tens of gigapascals to the terapascal regime [8, 9], in 1D (cylindrical or spherical) or 2D (Mach reflection) configurations. Here we employ converging compression waves to show that EOS measurements can reasonably be performed at pressures into the gigabar regime.

\section{EXPERIMENTAL CONFIGURATION}

At NIF, a large effort has been devoted to developing hohlraum platforms with exquisite spherical drive symmetry, in order to induce symmetric implosion of the thermonuclear fuel capsule. We propose to take advantage of this work by replacing the fuel capsule with a solid sample assembly of the same diameter $-2 \mathrm{~mm}-$ and driving a shock into it. Compared with capsule implosions, convergent compression waves are generally more stable, as perturbations from symmetry tend to damp out.

It is desirable to use a plastic ablator $(\mathrm{CH})$, as it couples relatively well to the hohlraum radiation, can be doped to absorb hard x-rays that would preheat the sample, and most sample materials of interest have a higher shock impedance than $\mathrm{CH}$ thus increasing the pressure in the sample.

Ignition hohlraum temperatures can reach 250$300 \mathrm{eV}$, which should induce ablation pressures of 20-30 TPa in CH. Using existing EOS, we have predicted the effect of convergence on the pressure of shock and ramp waves. For shocks, the pressure passes through $100 \mathrm{TPa}$ at a radius of $100-200 \mu \mathrm{m}$, which should allow multi-gigabar pressures to be explored with readily achievable radiographic resolutions of around $20 \mu \mathrm{m}$ (Fig. 1).

The principal diagnostic for the mechanical EOS is streaked x-ray radiography, using a laser-heated plasma backlighter. A complementary diagnostic will be $\mathrm{x}$-ray Thomson scattering, which uses spectroscopy of scattered $\mathrm{x}$-rays to deduce the compression, temperature and electron density $[10,11,12]$.

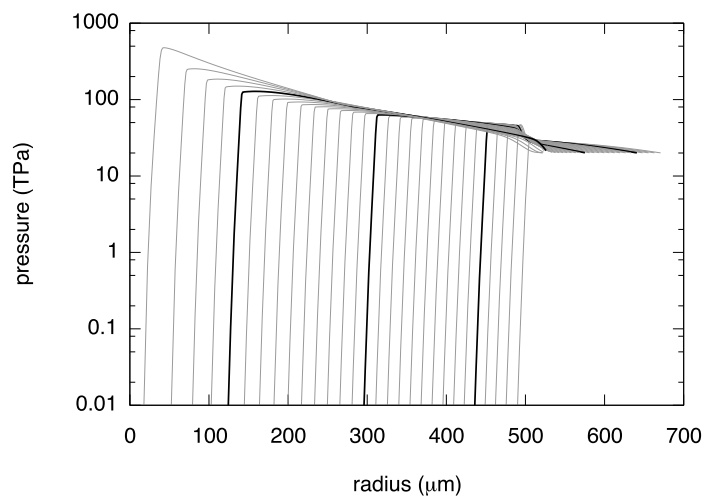

FIGURE 1. Pressure profiles for a diamond sample, driven using a $500 \mu \mathrm{m}$ thick $\mathrm{CH}$ ablator and a hohlraum temperature of $250 \mathrm{eV}$. Black lines: intervals of $1 \mathrm{~ns}$; grey lines: $0.1 \mathrm{~ns} .1$ gigabar is $100 \mathrm{TPa}$.

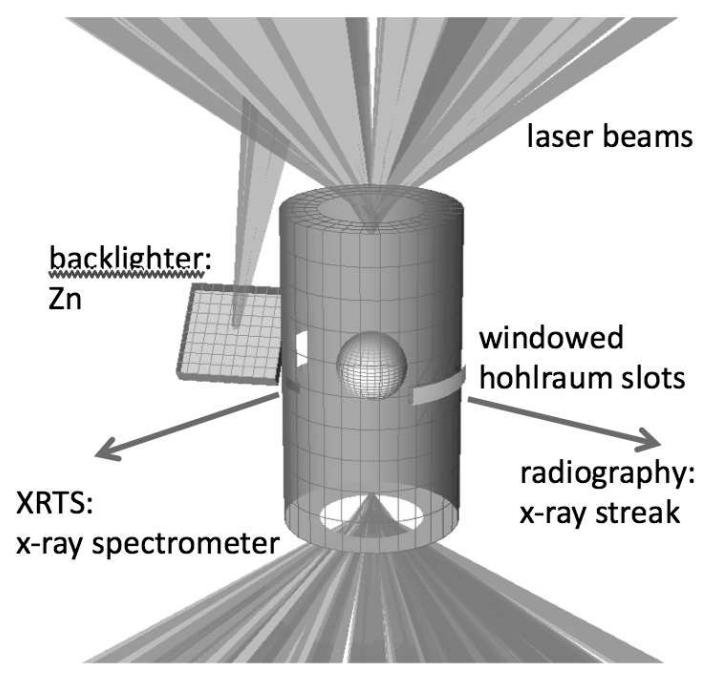

FIGURE 2. Experimental configuration.

He-like radiation from a $\mathrm{Zn}$ backlighter, at $8.95 \mathrm{keV}$, seems to be optimal for both diagnostics simultaneously, based on current backlighter development for NIF. Windows are needed in the hohlraum wall to allow the $\mathrm{x}$-rays to pass. It is most efficient to use windows no larger than necessary for the diagnostics, but the windows then act as a 3D source of cooling which makes full hohlraum simulations less tractable: we may use a cylindrical window to improve azimuthal and simulation symmetry. (Fig. 2.) 


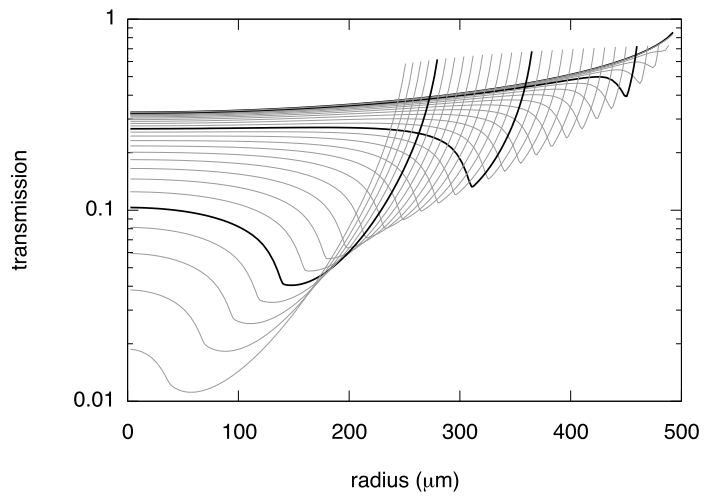

FIGURE 3. Simulated $x$-ray transmission through $1 \mathrm{~mm}$ diamond sample within $\mathrm{CH}$ ablator.

It would be valuable to obtain gigabar data on a variety of materials. For the initial experiments, it is desirable to choose a material that allows an accurate measurement to be made. One limiting factor is the $\mathrm{x}$-ray signal level, controlled by the sample opacity and thickness. Low density samples such as plastics are predicted to reach a high enough shock temperature that the opacity is likely to fall significantly from its initial value. Quantitative radiography would be possible only by introducing marker layers doped with a higher $Z$ : this greatly complicates sample fabrication, and the EOS of the doped material may be significantly different. Solid matter of $Z=13$ (Al) and higher generally remains cooler, but the x-ray transmission would be low. The transmission through the sample can be increased by decreasing its radius, using a much thicker ablator layer, at the cost of a smaller region reaching gigabar pressures. The best compromise found so far is to use a $\mathrm{C}$ (diamond) sample of $1 \mathrm{~mm}$ diameter, inside a $\mathrm{CH}$ sphere of $2 \mathrm{~mm}$ diameter. The smallest $\mathrm{x}$-ray transmissions are predicted to be several percent through the region exceeding $100 \mathrm{TPa}$ (Fig. 3).

If possible, additional radiographic images will be taken along the hohlraum axis, to measure azimuthal symmetry.

\section{OMEGA EXPERIMENTS}

A complementary series of experiments will be performed at the Omega laser facility, to develop and refine experimental and analysis methods. The hohlraum and sample will be smaller, the sample $0.6 \mathrm{~mm}$ in diameter. The first experiments will use a solid $\mathrm{CH}$ sample, and are expected to provide EOS data up to several tens of terapascals. The opacity of $\mathrm{CH}$ is predicted to remain close to its initial value over most of the range.

\section{RADIOGRAPHIC ANALYSIS}

EOS measurements from the converging shock proposed here require radiographic measurement of the shock speed and compression as a function of radius, which in turn require the location of the shock to be determined as a function of time and the density jump at that location. Unlike a supported shock in plane geometry, the density varies with radius behind the shock, so an adequate spatial resolution is needed to infer the value at the shock. It is not practical to capture the whole radial profile of the solid sample, ablated material from the sample and hohlraum wall, and the residual wall on the x-ray streak record, so it is not possible to perform an Abel inverse of the onion skin type. Further, any radiographic unfold process which works radially inward will accumulate errors. Fortunately, the unshocked region at the center of the sample provides a strong constraint on the unfold in the region of the shock, and the shape of the transmission profile in the center contains information about the attenuation further out. This information can be extracted by looking for long modes in the transmission profile [13].

An alternative approach is to use a parameterized Bayesian profile matching process, where the density in the central region is fixed. The position and amplitude of the shock, and the density profile further out, are described with adjustable parameters, which are optimized to give the best match to the attenuation profile.

We investigated the use of different algorithms, including Abel inversion and profile matching, for simulated radiographic data to which random noise was added. Abel inversion magnified the noise by over an order of magnitude around the radius of the shock. Profile matching was capable of reproducing the position and amplitude of the shock to within a few percent. The recostructed profile in the region of the shock was not sensitive to inaccuracies in 


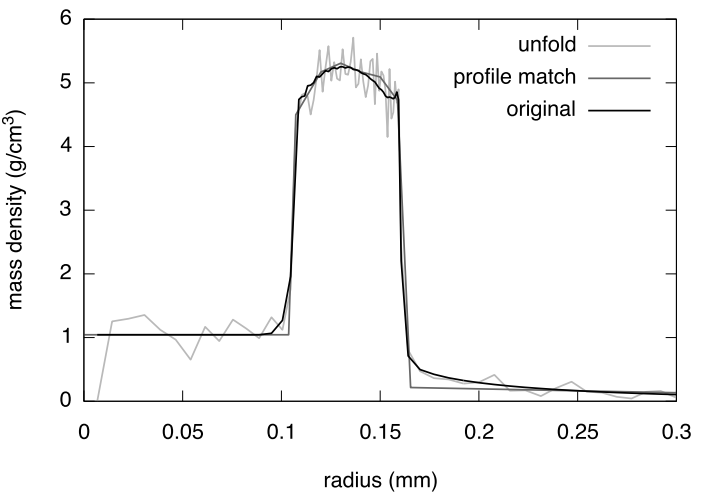

FIGURE 4. Unfolds of simulated radiographic data, for the initial Omega experiments on $\mathrm{CH}$, using different algorithms. The profile match was not allowed enough degrees of freedom to reproduce the low density region at $\sim 0.17 \mathrm{~mm}$ radius, but the reconstruction of the shock was still accurate.

reconstructing the profile in the outer, low density region. (Fig. 4)

Generally, the shock speed is expected to vary smoothly with radius, and the density profile behind the shock should also vary smoothly with time, so smoothing in the temporal direction can be used to reduce the noise in the unfolded density profiles. We estimate this smoothing to reduce the uncertainty in EOS measurements by a factor of several.

\section{CONCLUSIONS}

We have designed an experimental platform for NIF which should access gigabar pressures using hohlraum driven spherical samples, and streak radiography. Because of pulse length constraints and the need to base the design on thermonuclear ignition configurations in order to take advantage of the large effort on drive symmetry, ramp loading is not feasible initially. However, with a converging shock, radiographic measurements explore a range of states along the principal shock Hugoniot in each experiment. Radiographic measurement of the shock compression requires an accurate knowledge of the opacity of the sample, which means that the sample temperature must remain well below the energy of the electronic $K$ edge. For higher temperatures, radiographic marker layers could be used. We have de- veloped designs for the first series of experiments at NIF and OMEGA. Analysis so far suggests that accuracies $o(1 \%)$ should be achievable in shock speed and compression.

\section{ACKNOWLEDGMENTS}

We would like to thank Dr Damien Hicks (Lawrence Livermore National Laboratory) for advice on radiography of hohlraum-driven samples. This work was performed under the auspices of the U.S. Department of Energy under contract \# DE-AC52-07NA27344.

\section{REFERENCES}

1. Seager, S., Kuchner, M., Hier-Majumder, C.-A., and Militzer, B., Astrophys. J., 669, 1279-1297, 2007.

2. Swift, D. C., Eggert, J., Hicks, D.G., Hamel, S., Caspersen, K., Schwegler, E., Collins, G.W., Nettelmann, N., and Ackland, G.J., submitted (2011).

3. Bertone, G, Hooper, D, and Silk, J, Phys. Rep., 405, 5-6, 279, 2005.

4. Ragan III, C.E., Phys. Rev. A, 25, 3360-3375, 1982.

5. Avrorin, E.N., Vodolaga, B.K., Simonenko, V.A., and Fortov, V.E., Phys.-Uspekhi, 36, 5, 1993.

6. Collins, G.W., et al, Science, 281, 5380, 1178-1181, 1998.

7. Eggert, J., et al, these proceedings.

8. Kanzleiter, R.J., Atchison, W.L., Bowers, R.L., Fortson, R.L., Guzik, J.A., Olson, R.T., Stokes, J.L., and Turchi, P.J., IEEE Trans. Plasma Sci., 30, 5, 1755-1763, 2002.

9. Swift, D.C. and Ruiz, C.R., AIP Conf. Proc., 845, 1297-1300, 2006.

10. Kritcher, A.L., et al, Science, 322, 5898, 69-71, 2008.

11. Glenzer, S., and Redmer, R., Rev. Mod. Phys. 81, 4, $1625,2009$.

12. Kritcher, A.L., et al, Phys. Rev. Lett., 107, 015002, 2011. Phys. Rev. Lett. 107, 015002 (2011)

13. Hicks, D.G., et al, Phys. Plasmas, 17, 102703, 2010. 